\title{
Comparison and analysis of reoperations in two different treatment protocols for trochanteric hip fractures - postoperative technical complications with dynamic hip screw, intramedullary nail and Medoff sliding plate
}

Johnny Paulsson, Josefine Corin Stig and Ola Olsson * (1)

\begin{abstract}
Background: In treatment of unstable trochanteric fractures dynamic hip screw and Medoff sliding plate devices are designed to allow secondary fracture impaction, whereas intramedullary nails aim to maintain fracture alignment. Different treatment protocols are used by two similar Swedish regional emergency care hospitals. Dynamic hip screw is used for fractures considered as stable within the respective treatment protocol, whereas one treatment protocol (Medoff sliding plate/dynamic hip screw) uses biaxial Medoff sliding plate for unstable pertrochanteric fractures and uniaxial Medoff sliding plate for subtrochanteric fractures, the second (intramedullary nail/dynamic hip screw) uses intramedullary nail for subtrochanteric fractures and for pertrochanteric fractures with intertrochanteric comminution or subtrochanteric extension. All orthopedic surgeries are registered in a regional database.
\end{abstract}

Methods: All consecutive trochanteric fracture operations during 2011-2012 $(n=856)$ and subsequent technical reoperations $(n=40)$ were derived from the database. Reoperations were analysed and classified into the categories adjustment (percutaneous removal of the locking screw of the Medoff sliding plate or the intramedullary nail, followed by fracture healing) or minor, intermediate (reosteosynthesis) or major (hip joint replacement, Girdlestone or persistent nonunion) technical complications.

Results: The relative risk of intermediate or major technical complications was 4.2 (1.2-14) times higher in unstable pertrochanteric fractures and $4.6(1.1-19)$ times higher in subtrochanteric fractures with treatment protocol: intramedullary nail/dynamic hip screw, compared to treatment protocol: Medoff sliding plate/dynamic hip screw. Overall rates of intermediate and major technical complications in unstable pertrochanteric and subtrochanteric fractures were with biaxial Medoff sliding plate $0.68 \%$, with uniaxial Medoff sliding plate $1.4 \%$, with dynamic hip screw $3.4 \%$ and with intramedullary nail $7.2 \%$.

Conclusions: The treatment protocol based on use of biaxial Medoff sliding plate for unstable pertrochanteric and uniaxial Medoff sliding plate for subtrochanteric fractures reduced the risk of severe technical complications compared to using the treatment protocol based on dynamic hip screw and intramedullary nail.

Keywords: Pertrochanteric, Subtrochanteric, Fracture, Reoperation, Intramedullary nail, Medoff sliding plate, Dynamic hip screw

* Correspondence: ola.a.olsson@skane.se

Department of Orthopedics, Helsingborg Hospital, S-251 87 Helsingborg,

Sweden 


\section{Background}

Stable two-fragment pertrochanteric fractures are treated with good result with dynamic hip screw (DHS) devices $[1,2]$, but controversy remains regarding treatment of unstable pertrochanteric and subtrochanteric fractures. Dynamic fixation methods are designed to allow secondary fracture impaction to improve interfragmentary stress transfer, thereby facilitating fracture healing and unloading the implant. DHS allows dynamic compression along the axis of the femoral neck, which, depending on fracture geometry, may be more or less appropriate or effective [3, 4].

The Medoff sliding plate [5] (MSP) adds compression along the axis of the femoral shaft, thus allowing biaxial dynamisation. Lag screw sliding is optional and may be blocked by a locking screw to prevent medialisation of the femoral shaft, thereby creating uniaxial dynamisation.

Intramedullary nail (IMN) fixation is most commonly used worldwide for unstable pertrochanteric and subtrochanteric fractures and provides a strong and rigid fixation [6]. However, poor load transfer may subject the implant to high loads with increased risk of nonunion, implant failure or fracture due to stress concentration $[2,7]$.

In Skåne, Sweden, two similar regional emergency care hospitals use different treatment protocols for trochanteric fractures. For pertrochanteric fractures considered as stable both hospitals use DHS, whereas for fractures considered as unstable within the respective treatment protocol, Helsingborg Hospital uses MSP, and Kristianstad Hospital uses IMN. All orthopedic operations performed are consecutively registered in a mutual regional database and both hospitals cover similar population based catchment areas.
The aim of this consecutive retrospective study was to analyse and compare all performed reoperations and related technical complications in all pertrochanteric and subtrochanteric fractures, treated during two years according to two different treatment protocols in two parallel consecutive case series.

\section{Methods}

The two treatment protocols for choice of fixation methods (MSP/DHS or IMN/DHS) are described in Table 1. In short, DHS was used in fractures considered as stable, whereas treatment protocol: MSP/DHS aimed to use MSP in all pertrochanteric fractures with 3 or more fragments and treatment protocol: IMN/DHS aimed to use IMN in fractures with intertrochanteric comminution or fracture extension below the trochanters. In subtrochanteric fractures treatment protocol: MSP/DHS used locked MSP and treatment protocol: IMN/DHS used IMN. The implants used were the Medoff sliding plate (MSP) or Hansson plate (DHS) (Swemac Orthopaedics, Linköping, Sweden), or IM nails (Gamma Nail (Stryker, Kalamazoo, MI, USA), Intertan and Trochanteric Anterior Nail (Smith\&Nephew, Memphis, TN, USA). The DHS and MSP were combined with either a Twin hook [8] ( $n=697$ ) (Swemac, Orthopaedics, Linköping, Sweden) or a lag screw $(n=5)$. The Twin hook wings may be retracted, which allowed percutaneous technique with the plate left in situ when adjustment, replacement or removal of the Twin hook was needed. The MSP was used in biaxial or uniaxial mode (locking screw for the Twin hook/ lag screw). In treatment protocol: MSP/DHS repeat radiographs were obtained 10 days postoperatively when MSP was used in uniaxial mode. If axial sliding capacity of the plate was consumed $(25 \mathrm{~mm})$, or migration of the Twin

Table 1 Treatment protocols for pertrochanteric and subtrochanteric fractures in the two treatment groups

\begin{tabular}{|c|c|c|}
\hline \multirow[t]{2}{*}{ Fracture type } & \multicolumn{2}{|l|}{ Fixation method } \\
\hline & Treatment protocol: MSP/DHS ${ }^{a}$ & Treatment protocol: IMN/DHS ${ }^{\mathrm{b}}$ \\
\hline \multicolumn{3}{|l|}{ Pertrochanteric } \\
\hline Stable - 2 fragments ${ }^{c}$ & DHS & \\
\hline Unstable - 3 or more fragments ${ }^{c}$ & MSP biaxial & \\
\hline Unstable - no lateral and/or no posterior support ${ }^{c}$ & MSP uniaxial (locked) & \\
\hline Stable & & DHS \\
\hline Intertrochanteric comminution and/or subtrochanteric extension ${ }^{d}$ & & IMN \\
\hline Subtrochanteric & MSP uniaxial (locked) & IMN \\
\hline Fracture extension below $>2$ of & IMN or & \\
\hline MSP plate screws ${ }^{\mathrm{e}}$ & long DHS & \\
\hline
\end{tabular}

${ }^{a}$ Medoff sliding plate (MSP) / dynamic hip screw (DHS)

bIntramedullary nail (IMN) / dynamic hip screw (DHS)

${ }^{c}$ Choice of treatment based on Jensen Michaelsen and Seinsheimer classifications including analysis of lateral and posterior support

${ }^{\mathrm{d} C h o i c e ~ o f ~ t r e a t m e n t ~ b a s e d ~ o n ~ i n t e r t r o c h a n t e r i c ~ f r a c t u r e ~ c o m m i n u t i o n ~ o r ~ s u b t r o c h a n t e r i c ~ f r a c t u r e ~ e x t e n s i o n ~}$

${ }^{\mathrm{e}}$ Approximately $10 \mathrm{~cm}$ below the lesser trochanter 
hook/lag screw was observed, the locking screw was routinely removed to allow biaxial dynamisation.

From the mutual regional orthopedic surgery database all orthopedic operations performed in the two Skåne regional hospitals during 2011 and 2012 were assessed. Data on all operations with a surgical procedure code for plate or intramedullary nail, and any of the International Code of Disease (ICD-10) codes S7200/01 (femoral neck fracture), S7210/11 (pertrochanteric fracture) or S7220/21 (subtrochanteric fracture), were retrieved $(n=892)$. The total number of previously performed trochanteric hip fracture surgeries was also derived from the database for each individual non-specialist surgeon.

All fractures were classified by evaluation of available pre-, intra- and postoperative radiographs. Following exclusion of femoral neck and basicervical fractures, a final study group of 856 patients was set (Fig. 1).
Pertrochanteric fractures were classified according to Jensen Michaelsen (JM) [9], and subtrochanteric fractures according to Seinsheimer (SH) [10]. In the study analysis JM types 1-2 (2 fragments) were considered as stable and JM types 3-4-5 (3 or more fragments) and all subtrochanteric fractures as unstable.

Fractures were also classified regarding lateral and/or posterior support, defined as the presence of intact cortical bone of the femoral shaft at the level of or above the center of the lag screw entry hole.

Fracture classification was performed by a single examiner. To assess interobserver variability 75 randomly selected fractures were also classified by a second independent observer.

To find relevant reoperations all additional orthopedic operations performed in any Skåne regional hospital after the index operation on the 856 study patients between

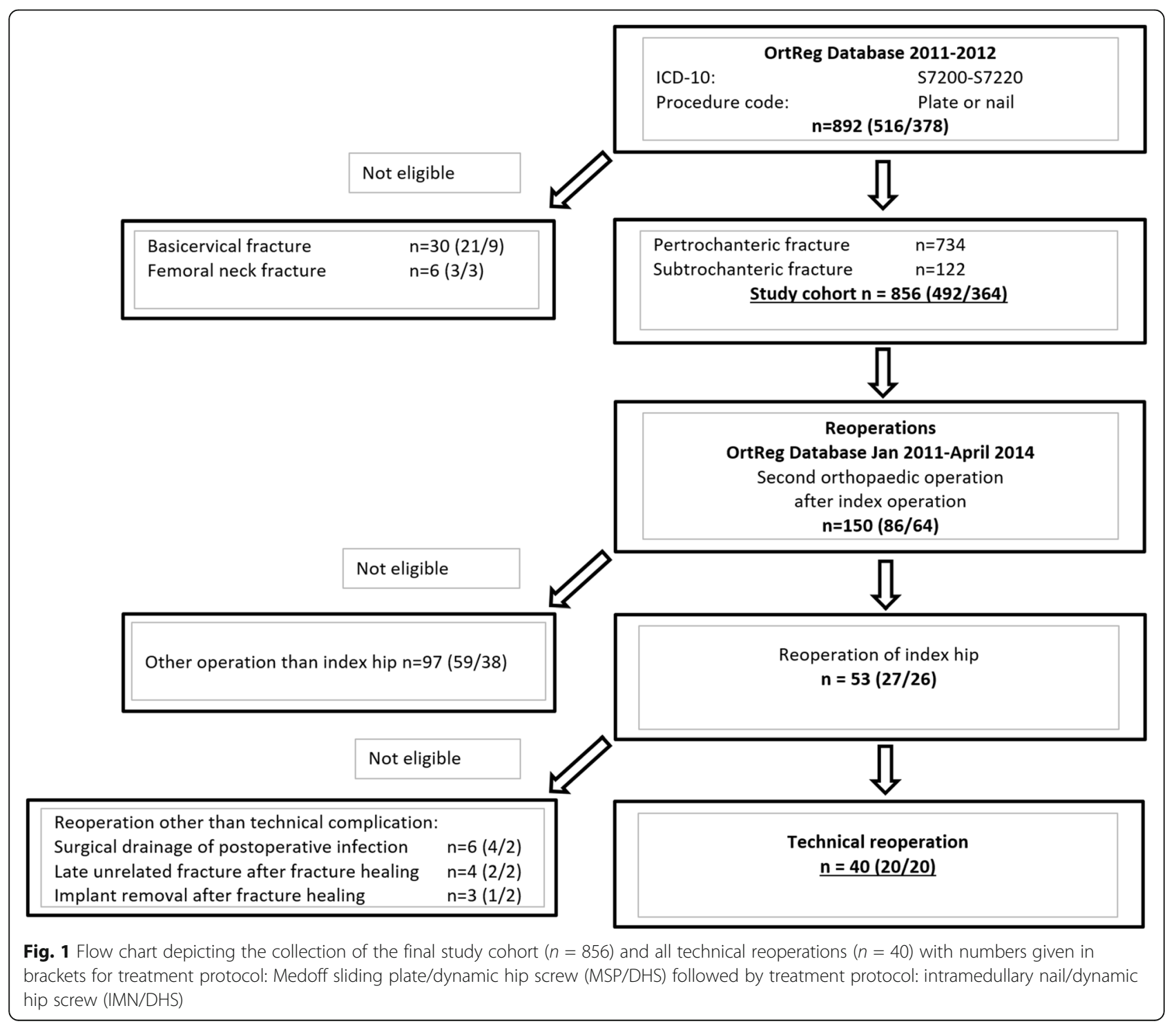


January 2011 and April 2014, thus with a follow-up time span from 16 to 40 months, were derived from the regional database. After exclusion of other orthopedic surgical procedures and of reoperations other than technical, a final total of 40 technical reoperations was set (Fig. 1). For these cases, technical reoperations and the postoperative course of events were analysed in detail using all available postoperative radiographs and medical charts. Quality of fracture reduction and implant positioning was classified as good, acceptable or poor [11-13] and definitions were set to analyse reoperations, final outcome and related technical complications in the four categories adjustment (removal of locking screw, no technical complication) or minor (percutaneous withdrawal, replacement or removal of Twin Hook/lag screw due to penetration, followed by fracture healing), intermediate (reosteosynthesis due to non-union, femoral shaft stress fracture or varisation/cut-out) or major (loss of hip joint integrity with Girdlestone procedure or hip joint replacement, or persistent nonunion).

\section{Statistical analysis}

Statistical analyses were made using Analyse-it Standard Edition 3.20 (Analyse-it Software, Ltd. Leeds, UK). Median was given with 25 th and 75 th percentiles. Comparisons between groups were made using the chi-squared test or by calculation of relative risk (RR) with 95\% C.I. Results with a $p<0.05$ were considered as significant.

\section{Results}

\section{Study group}

The treatment protocol: MSP/DHS $(n=492)$ and treatment protocol: IMN/DHS $(n=364)$ groups were similar regarding age, gender, distribution of different fracture subtypes, number of surgeons and surgeons experience. Median age was 85 (79-89) years and two thirds of the patients were female. The operations were made by a surgeon having performed 25 or more previous trochanteric hip fracture surgeries in $68 \%$ of the patients in treatment protocol MSP/DHS and in $87 \%$ in treatment protocol IMN/DHS, respectively. $16 \%$ of all fractures were classified as stable pertrochanteric, 70\% unstable pertrochanteric and 14\% subtrochanteric. Lack of lateral support was noted in $19 \%$ and of posterior support in $48 \%$ of all fractures.

In assessment of interobserver variability, classification agreement was $75 \%(56 / 75)$ for specific JM/SH fracture subtypes, whereas $97 \%$ (73/75) were uniformly classified as stable or unstable.

\section{Fixation methods}

A vast majority of stable pertrochanteric fractures were treated using DHS in both treatment groups. Unstable pertrochanteric fractures, according to study fracture classification, were operated with MSP in biaxial mode $(82 \%)$ or in uniaxial mode $(9 \%)$ in treatment protocol: MSP/DHS. In 9\% DHS was used, not compliant with the treatment protocol. In treatment protocol: IMN/DHS $35 \%$ were operated with IMN and 65\% with DHS, but of all fractures JM types 3-4-5 only 2\% (6/251) had lack of lateral support and were treated with DHS, contradicting the treatment protocol. Subtrochanteric fractures were treated using IMN (92\%) or DHS (8\%) in treatment protocol: IMN/DHS, and with MSP in uniaxial (61\%) or biaxial mode (7\%), with IMN (23\%) or with DHS (9\%) in treatment protocol: MSP/DHS (Table 2).

\section{Treatment groups and reoperations}

There was a total of 40 technical reoperations in 856 patients, 20 in treatment protocol: MSP/DHS (4.1\%, Table $3)$ and $20(5.5 \%)$ in treatment protocol: IMN/DHS $(p=0.33$, Table 4$)$. Quality of fracture reduction and of implant positioning in the 40 reoperated patients appeared to be similar in the 2 treatment groups (Tables 3 and 4). 13 technical complications (2.6\%) occurred in treatment protocol: MSP/DHS and 19 (5.2\%) in treatment protocol: IMN/DHS $(p=0.049)$. The rate of intermediate and major complications was more than 3 times higher (RR 3.6, 1.5-8.9) in treatment protocol: IMN/ DHS $(16 / 364,4.4 \%)$ than in treatment protocol: MSP/ DHS $(6 / 492,1.2 \%)(p=0.0037)$. Documented absence of fracture healing (joint replacement, Girdlestone procedure or persisting nonunion) occurred in 10 cases in treatment protocol: IMN/DHS (2.7\%) and in 3 cases $(0.61 \%)$ in treatment protocol: MSP/DHS $(p=0.011$, $\mathrm{RR}=4.5,1.3-15$ ) (Fig. 2).

Stable pertrochanteric fractures had a total reoperation rate of $0.75 \%$, unstable pertrochanteric $3.2 \%$ and subtrochanteric $16 \%$. Intermediate and major technical complications were more than 4 times more frequent in treatment protocol: IMN/DHS than in treatment protocol: MSP/DHS in both unstable pertrochanteric fractures $(p=0.018)$ and in subtrochanteric fractures $(p=0.031$, Table 5).

\section{Implants and reoperations}

Total reoperation rate with biaxial MSP was $1.3 \%$, with DHS 3.1\% and with IMN 8.4\% (Fig. 3). With MSP in uniaxial mode 13 of 74 (18\%) were reoperated, 92\% of these were adjustments $(n=7)$ or minor technical complications $(n=5)$. Overall rate of intermediate and major technical complications in all unstable pertrochanteric and subtrochanteric fractures was with uniaxial MSP 1.4\% and was lower with biaxial MSP $(0.68 \%)$ than with IMN $(7.2 \%$, $p=0.0001)$ and with DHS $(3.4 \%, p=0.023)$. For unstable pertrochanteric fractures separately rate of intermediate and major technical complications was lower with biaxial MSP $(0.69 \%)$ than with IMN $(4.5 \%, p=0.011)$, and was 
Table 2 Number of fractures, and fraction within fracture type group, treated with each fixation method for different fracture subtypes in the 2 treatment groups

\begin{tabular}{|c|c|c|c|c|c|c|}
\hline \multirow[t]{2}{*}{ Fracture type ${ }^{c}$} & \multicolumn{4}{|c|}{ Treatment protocol: MSP/DHS ${ }^{a}$} & \multicolumn{2}{|c|}{ Treatment protocol: IMN/DHS } \\
\hline & DHS & MSP biaxial & MSP locked uniaxial & IMN & DHS & IMN \\
\hline Stable pertrochanteric & $56(77 \%)$ & $17(23 \%)$ & 0 & 0 & $59(98 \%)$ & $1(2 \%)$ \\
\hline JM1 & $28(88 \%)$ & $4(12 \%)$ & & & $39(98 \%)$ & $1(2 \%)$ \\
\hline $\mathrm{JM} 2$ & $28(68 \%)$ & $13(32 \%)$ & & & $20(100 \%)$ & 0 \\
\hline Unstable pertrochanteric & $30(9 \%)$ & $288(82 \%)$ & $32(9 \%)$ & 0 & $163(65 \%)$ & $88(35 \%)$ \\
\hline JM3 & $13(16 \%)$ & $60(74 \%)$ & $8(10 \%)$ & & $67(89 \%)$ & $8(11 \%)$ \\
\hline JM4 & $12(20 \%)$ & $43(73 \%)$ & $4(7 \%)$ & & $13(59 \%)$ & $9(41 \%)$ \\
\hline JM5 & $5(2 \%)$ & $185(88 \%)$ & $20(10 \%)$ & & $83(54 \%)$ & $71(46 \%)$ \\
\hline Subtrochanteric & $6(9 \%)$ & $5(7 \%)$ & $42(61 \%)$ & $16(23 \%)$ & $4(8 \%)$ & $49(92 \%)$ \\
\hline \multicolumn{7}{|l|}{ Divergence from treatment protocol $^{d}$} \\
\hline $\begin{array}{l}\text { JM1-JM2 with no lateral or no posterior support } \\
\text { or JM3-JM4-JM5 }\end{array}$ & $31\left(1^{\mathrm{e}}\right)$ & & & & & \\
\hline JM 3-4-5 with no lateral support & & & & & $6\left(2^{\mathrm{e}}\right)$ & \\
\hline $\begin{array}{l}\text { JM3-JM4-JM5 with no lateral or no posterior } \\
\text { support or subtrochanteric }\end{array}$ & & $8\left(0^{\mathrm{e}}\right)$ & & & & \\
\hline
\end{tabular}

with DHS 3.1\% and with uniaxial MSP none (Table 5). In 42 subtrochanteric fractures operated with uniaxial MSP one intermediate technical complication (2.4\%) occurred and in 65 operated with IMN 7 intermediate or major technical complications occurred $(11 \%, p=0.11)$.

\section{Lateral/posterior support}

In total, 5 reoperations (1.6\%) were performed in 308 unstable pertrochanteric fractures with intact lateral and posterior support. Of the 4 technical complications 3 were minor and only one $(0.32 \%)$ was major. In the 293 unstable pertrochanteric fractures with lack of lateral or posterior support $14(4.8 \%)$ reoperations and technical complications occurred, 11 (3.8\%) of these intermediate $(n=3)$ or major $(n=8,2.7 \%)$. Lack of posterior support was more frequent than lack of lateral support, but only one of the 14 reoperations had intact posterior support compared to 10 with intact lateral support.

\section{Implants and lateral/posterior support}

In unstable pertrochanteric fractures with intact lateral and posterior support, reoperation rates were with DHS 2/136 (1.5\%), with biaxial MSP $1 / 143$ (0.70\%) and none of the 24 fractures operated with IM. If either lateral or posterior support also was lacking, 6 (11\%) technical complications occurred with DHS, 5 of these intermediate $(n=1)$ or major $(n=4,7.0 \%)$ and with biaxial MSP $2.1 \%(3 / 145, p=0.0063)$, one intermediate and one $(0.70 \%)$ major. All reoperations with IMN in unstable pertrochanteric fractures $(4 / 88,4.5 \%)$ lacked lateral or posterior support.

\section{Discussion}

The major finding in this study was the more than 4 times higher rate of technical complications with loss of hip integrity, persistent non-union or need for revision osteosynthesis, following treatment of both unstable pertrochanteric and subtrochanteric fractures according to treatment protocol: IMN/DHS compared to treatment protocol: MSP/DHS. The low technical complication rate with MSP in both unstable pertrochanteric and subtrochanteric fractures is in line with results reported in previous studies $[4,12,14-16]$. The main mechanisms behind major technical complications were malalignment with varus dislocation and cut-out due to anteromedialisation of the femoral shaft in fractures without lateral or posterior support operated with DHS or biaxial MSP, varus dislocation and cut-out in fractures treated with DHS or IMN due to insufficient load-sharing, or bone or implant stress fracture or nonunion due to load-bearing in fractures treated with IMN.

The retrospective study design was observational using post-hoc analysis of existing data, which usually provides less solid information due to limited control of systematic and consecutive study inclusion and incomplete outcome data. However, the regional orthopedic surgery database structure warranted complete consecutive inclusion of all operated patients as well as complete registration of the 


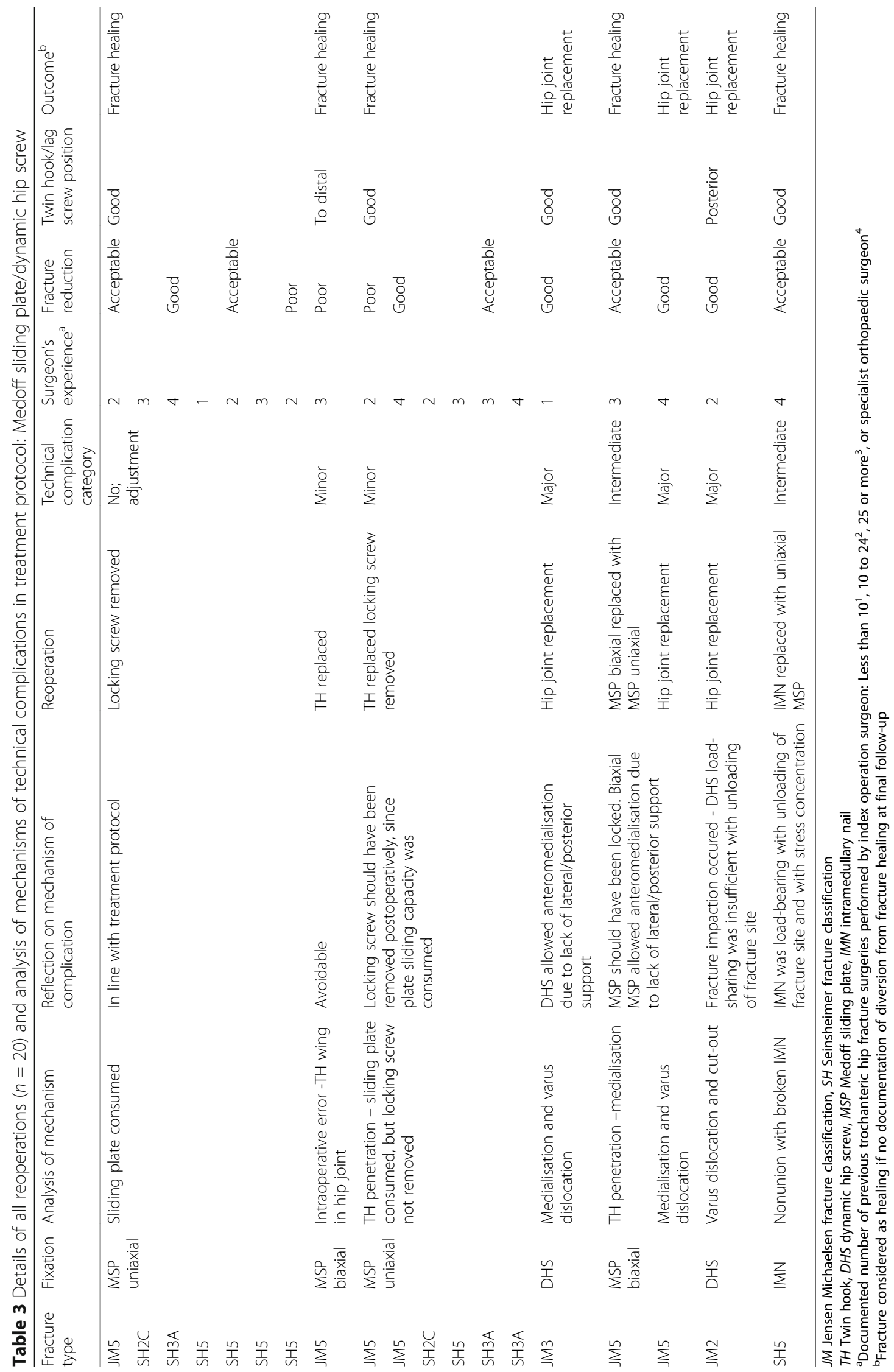




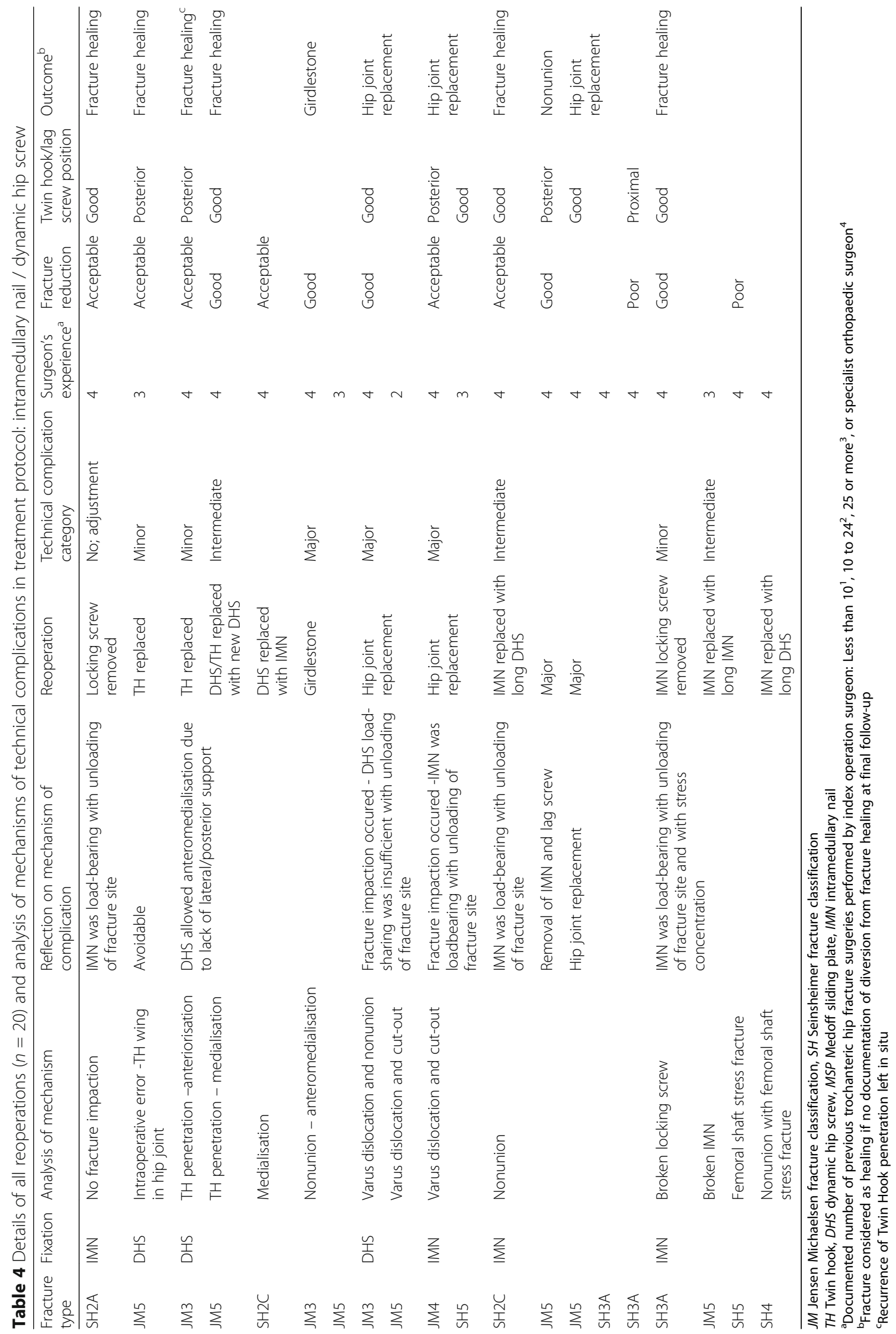




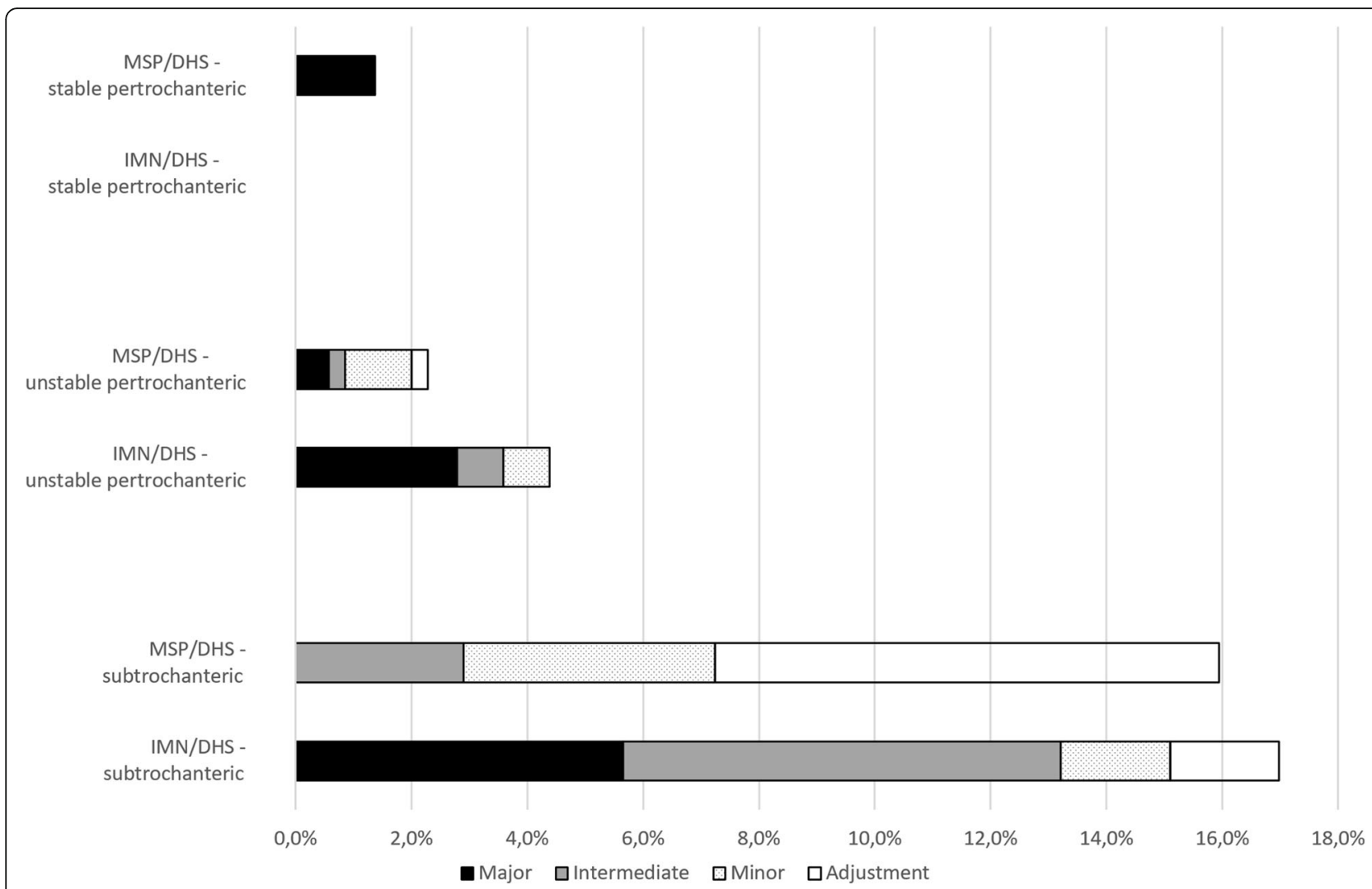

Fig. 2 Rates of adjustment reoperations and the different categories of reoperations with technical complications for stable pertrochanteric, unstable pertrochanteric and subtrochanteric fractures in treatment protocol: Medoff sliding plate/dynamic hip screw (MSP/DHS) and in treatment protocol: intramedullary nail/dynamic hip screw (IMN/DHS)

sole outcome parameter reoperation. Therefore, the study design may actually be considered a strength, since the objective of studying treatment protocol performance in regular daily care was not biased by study design, or by awareness among involved surgeons of an ongoing study. Systematic repeat radiographs after the postoperative examination were obtained only when uniaxial MSP was used. Reported rates of technical complications for the treatment protocols should therefore be considered as lower limits of true rates.

Assumed treatment group similarity to allow group comparisons appeared justified, since all fractures were consecutively included and no relevant group differences were observed regarding patient baseline data, fracture type distribution or surgical performance. Still, in addition to implants, existence of other group differing factors with possible impact on outcome, such as fracture pattern distribution or hospital specific performance, cannot be completely ruled out. Classification reliability was assessed by interobserver comparison and agreement was high, in particular in the important distinction between stable and unstable fractures.

Similar to other reports $[2,4,14]$, the main technical complications with DHS in unstable pertrochanteric fractures were varus dislocation with lag screw cut-out or nonunion, frequently related to femoral shaft medialisation. With MSP in biaxial mode, lower rate of fixation failure in unstable pertrochanteric fractures was reproduced in this study. The sliding mechanism of both the DHS and the MSP lag screw barrel is aligned parallel to the femoral neck. Hence, compression along this axis will induce anteromedial translation of the femoral shaft relative to the head and neck fragment, which may compromise stable contact and load transfer between the main proximal and distal fracture fragments. A sevenfold increase of technical fixation failures with DHS has been reported with a medialisation of $30 \%$ or more [17]. As with DHS, the main mechanism behind major complications with biaxial MSP is femoral shaft anteromedialisation, which however appeared to be less frequent and less pronounced than with DHS in this study as well as in others $[4,16]$, probably due to facilitated axial fracture impaction and interdigitation of fracture fragments. Still, as with DHS, biaxial MSP may be inappropriate if the fracture extends below the level of the lag screw entry hole with subsequent loss of lateral and or posterior support. Fracture malalignment has severe consequences and the vast majority of reoperations related to femoral 
Table 5 Number of technical complications in unstable pertrochanteric and in subtrochanteric fractures in the 2 treatment groups

\begin{tabular}{|c|c|c|c|c|}
\hline \multirow[t]{2}{*}{ Fracture type } & \multirow[t]{2}{*}{ Implant } & \multicolumn{2}{|c|}{ Treatment protocol } & \multirow[t]{2}{*}{ Relative risk ${ }^{c}$} \\
\hline & & $\overline{M S P} / \mathrm{DHS}^{\mathrm{a}}$ & $\mathrm{IMN}_{\mathrm{D}} \mathrm{DHS}^{\mathrm{b}}$ & \\
\hline \multirow[t]{18}{*}{ Unstable pertrochanteric } & & \multicolumn{2}{|c|}{ All technical complications } & \\
\hline & & $7 / 350(2,0 \%)$ & $11 / 251(4,4 \%)$ & \multirow[t]{5}{*}{$2.9(0.89-5.4)$} \\
\hline & DHS & $1 / 30$ & $7 / 163$ & \\
\hline & MSP biaxial & $4 / 288$ & - & \\
\hline & MSP uniaxial & $2 / 32$ & - & \\
\hline & IMN & - & $4 / 88$ & \\
\hline & & \multicolumn{2}{|c|}{ Intermediate and major technical complications } & \\
\hline & & $3 / 350(0,86 \%)$ & $9 / 251(3,6 \%)$ & \multirow[t]{5}{*}{$4.2(1.2-14)$} \\
\hline & DHS & $1 / 30$ & $5 / 163$ & \\
\hline & MSP biaxial & $2 / 288$ & - & \\
\hline & MSP uniaxial & $0 / 32$ & - & \\
\hline & IMN & - & $4 / 88$ & \\
\hline & & \multicolumn{2}{|c|}{ Major technical complications } & \\
\hline & & $2 / 350(0,57 \%)$ & $7 / 251(2,8 \%)$ & \multirow[t]{5}{*}{$4.9(1.2-21)$} \\
\hline & DHS & $1 / 30$ & $4 / 163$ & \\
\hline & MSP biaxial & $1 / 288$ & - & \\
\hline & MSP uniaxial & $0 / 32$ & - & \\
\hline & IMN & - & $3 / 88$ & \\
\hline \multirow[t]{18}{*}{ Subtrochanteric } & & \multicolumn{2}{|c|}{ All technical complications } & \\
\hline & & $5 / 69(7,2 \%)$ & $8 / 53(15 \%)$ & \multirow[t]{5}{*}{$2.1(0.78-5.8)$} \\
\hline & DHS & $0 / 6$ & $1 / 4$ & \\
\hline & MSP biaxial & $0 / 5$ & - & \\
\hline & MSP uniaxial & $4 / 42$ & - & \\
\hline & IMN & $1 / 16$ & $7 / 49$ & \\
\hline & & \multicolumn{2}{|c|}{ Intermediate and major technical complications } & \\
\hline & & $2 / 69(2,9 \%)$ & $7 / 53(13 \%)$ & \multirow[t]{5}{*}{$4.6(1.1-19)$} \\
\hline & DHS & $0 / 6$ & $1 / 4$ & \\
\hline & MSP biaxial & $0 / 5$ & - & \\
\hline & MSP uniaxial & $1 / 42$ & - & \\
\hline & IMN & $1 / 16$ & $6 / 49$ & \\
\hline & & \multicolumn{2}{|c|}{ Major technical complications } & \\
\hline & & $0 / 69$ & $3 / 53(5,7 \%)$ & \multirow[t]{5}{*}{$8.6(0.45-163)$} \\
\hline & DHS & $0 / 6$ & $0 / 4$ & \\
\hline & MSP biaxial & $0 / 5$ & 0 & \\
\hline & MSP uniaxial & $0 / 42$ & 0 & \\
\hline & IMN & $0 / 16$ & $3 / 49$ & \\
\hline
\end{tabular}

DHS dynamic hip screw, MSP Medoff sliding plate, IMN intramedullary nail

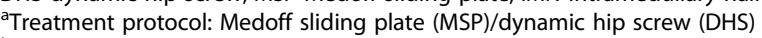

${ }^{\mathrm{b}}$ Treatment protocol: intramedullary nail (IMN)/dynamic hip screw (DHS)

${ }^{\mathrm{c}} 95 \% \mathrm{Cl}$

shaft anteromedialisation in this study were associated with major complications. According to treatment protocol: MSP/DHS MSP should be used in uniaxial mode in the absence of lateral or posterior support. This strategy prompted some adjustment procedures to allow secondary dynamisation, or minor reoperations due to Twin hook/lag screw penetration, but no major complications occurred.

The IMN maintains alignment and prevents femoral shaft anteromedialisation. Still, due to poor load transfer from implant to fracture site, varus dislocation and 


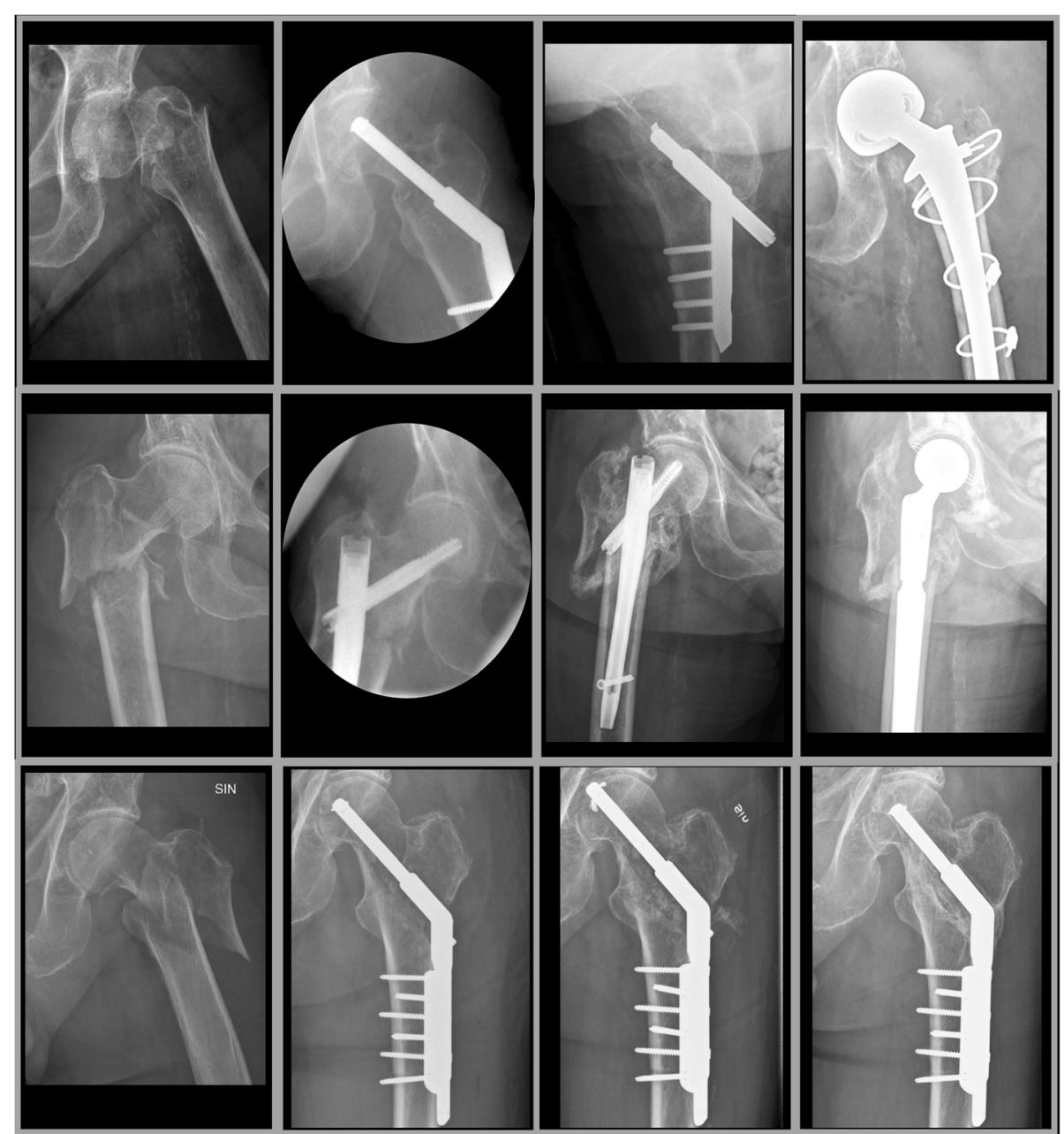

Fig. 3 From left to right preoperative, intra/postoperative, post technical complication and final radiographs of the typical and most frequent type of technical complication that occurred with dynamic hip screw (medialisation and nonunion followed by hip joint replacement, upper panels), intramedullary nail (nonunion followed by hip joint replacement, middle panels) and uniaxial Medoff sliding plate (Twin hook penetration followed by Twin hook replacement and fracture healing, lower panels)

cut-out may occur following loss of fixation purchase in the femoral head and neck fragment $[13,18]$. If purchase is maintained, nonunion, implant breakage or femoral shaft stress fracture may occur due to persistent unloading of the fracture surfaces [19-22], typical complications that were all observed in the present study.

In subtrochanteric fractures lateral and/or posterior support is by definition always absent. Miedel [23] reported reoperations in subtrochanteric fractures operated with MSP due to medialisation. However, in this study the MSP was used in biaxial mode. In subtrochanteric fractures MSP should always be used in uniaxial mode $[24,25]$ to achieve efficient load-sharing and prevent malalignment, at the expense of need for secondary dynamisation in a minority of patients to avoid risk of Twin hook/lag screw penetration. Nevertheless, as noted in this and in other studies [26, 27], with MSP in uniaxial mode Twin hook/lag screw penetration should not be expected to be completely avoided. With maintained axial and varus/valgus alignment this complication is however less demanding and may be resolved with a minor procedure, particularly with the Twin hook allowing percutaneous adjustments. Compared to the MSP, the IMN had a higher rate of intermediate and major technical complications, all related to load-bearing, when used in subtrochanteric fractures.

In treatment protocol: MSP/DHS the aim was to use MSP in all cases with identification of one or more separate trochanteric fragment, that is all fractures types JM 3-4-5. In treatment protocol: IMN/DHS on the other hand, most of the intermediate unstable pertrochanteric fractures, retrospectively classified as JM3-4-5, but with intact lateral and posterior support were, in accordance 
with the treatment protocol, treated with DHS. Thus, as opposed to in treatment protocol: MSP/DHS, almost two thirds of pertrochanteric fractures with more than 2 main fragments were operated with DHS. The remaining third operated with IMN therefore most likely represents more complex fractures within these subgroups and implant specific complication rates cannot be directly compared in fractures with the same postoperative classification in the two treatment groups. Accordingly, group comparisons in this study refer to complete fracture type groups. It should also be pointed out that the treatment protocol: IMN/DHS pattern of chosen fixation methods is representative of the overall Swedish national use of fixation methods for pertrochanteric as well as subtrochanteric fractures [28].

The complication rate with DHS was almost as high as with the IMN, suggesting that treatment of mild to moderately complex unstable pertrochanteric fractures with DHS was not optimal. A more than sevenfold increase of the technical complication rate was observed in unstable pertrochanteric fractures treated with DHS, if in addition lack of lateral or posterior support was noted. Whether the intermediate unstable pertrochanteric fracture category would do better with IMN than with DHS cannot be answered by this study. Anteromedialisation due to lack of lateral/posterior support would be controlled by the IMN, but increasing issues related to load-bearing with stress concentration and non-union should be expected. Shortcomings in fracture classification firmly defining these fracture type subgroups limit the value of available studies, as does the fact that most fractures in this subgroup are probably not treated with IMN.

In treatment protocol: MSP/DHS, a vast majority of unstable pertrochanteric fractures were operated with biaxial MSP, which in terms of minimising risk of reoperation and technical complication appears superior to both alternatives for this spectrum of fracture types. Due to surgeons' assessment of lack of lateral or posterior support, 9\% of unstable pertrochanteric fractures in treatment protocol: MSP/DHS were treated with MSP in uniaxial mode. Among these, 2 lag screw penetrations occurred but no major complications, again supporting priority of alignment control over biaxial dynamisation in appropriate fracture patterns. From analysis of mechanisms of technical complications in this study as well as others [29], fractures at increased risk of reoperation can often be identified if additional parameters such as lateral and posterior support are examined.

Reoperations with minor complications had no documented divergence from final fracture healing and were by definition performed percutaneously. This type of technical complication per se should have minor consequences for most patients. Intermediate and major technical complications should be expected to have more profound consequences and these subgroups comprise the cases that should be considered true failures from a technical orthopedic surgical point of view.

\section{Conclusions}

In summary, in this consecutive retrospective observational study comparing two different treatment protocols, technical complications in unstable pertrochanteric and subtrochanteric fractures were less frequent and less severe with a treatment protocol based on MSP fixation, compared to a treatment protocol based on IMN fixation. In this respect, a vast majority of unstable pertrochanteric fractures are best treated with MSP in biaxial mode and stand greater risk of major complications if treated with DHS, due to loss of alignment or insufficient load-sharing, or with IMN due to load-bearing. In the most challenging unstable pertrochanteric and subtrochanteric fractures proper application of the MSP in uniaxial mode, in combining maintained alignment and effective load-sharing, will minimise the risk of major technical complications compared to DHS and IMN.

\section{Abbreviations \\ DHS: Dynamic hip screw; IMN: Intramedullary nail; JM: Jensen \& Michaelsen classification; MSP: Medoff sliding plate; SH: Seinsheimer classification; TH: Twin hook}

\section{Acknowledgements}

Not applicable.

\section{Funding}

This study was supported by grants from Stig and Ragna Gorthon Foundation, Helsingborg, Sweden. The funding body had no role in the design or in the conduction of the study.

\section{Availability of data and materials}

The datasets used during the current study are not publicly available due to patient integrity, but are available from the corresponding author on reasonable request.

\section{Authors' contributions}

$\mathrm{OO}$ and JP designed the study. JCS and JP collected all data. Data analysis was performed by JCS and JP under supervision of OO. Classification of radiographs were performed by JCS under supervision of $\mathrm{OO}$ and interobserver variability was assessed for JCS and OO. All authors contributed in reviewal and interpretation of data. The manuscript was drafted by JCS, reviewed by all authors and revised with contributions from all authors under supervision and final revision of $\mathrm{OO}$. $\mathrm{OO}$ was responsible for the integrity of the work from inception to finished article. All authors read and approved the final manuscript.

\section{Ethics approval and consent to participate}

This study was approved by the Lund University Ethics Committee, registration number 2015/345.

Patient consent to participate was not applicable due to study design, only involving retrospective review of orthopedic surgery database, radiograhs and medical charts.

Consent for publication

Not applicable. 


\section{Competing interests}

The authors declare that they have no competing interests. No benefits in any form have been received or will be received from a commercial party related directly or indirectly to the subjects of this article.

\section{Publisher's Note}

Springer Nature remains neutral with regard to jurisdictional claims in published maps and institutional affiliations.

Received: 20 April 2017 Accepted: 15 August 2017

Published online: 24 August 2017

\section{References}

1. Jensen JS, Sonne-Holm S, Tondevold E. Unstable trochanteric fractures. A comparative analysis of four methods of internal fixation. Acta Orthop Scand. 1980;51:949-62

2. Matre K, Havelin LI, Gjertsen JE, Espehaug B, Fevang JM. Intramedullary nails result in more reoperations than sliding hip screws in two-part intertrochanteric fractures. Clin Orthop Relat Res. 2013;471:1379-86.

3. Kyle RF, Wright TM, Burstein AH. Biomechanical analysis of the sliding characteristics of compression hip screws. J Bone Joint Surg Am. 1980;62:1308-14.

4. Olsson O, Ceder L, Hauggaard A. Femoral shortening in intertrochanteric fractures. A comparison between the Medoff sliding plate and the compression hip screw. J Bone Joint Surg Br. 2001;83:572-8.

5. Medoff RJ, Maes K. A new device for the fixation of unstable pertrochanteric fractures of the hip. J Bone Joint Surg Am. 1991;73:1192-9.

6. Matre K, Havelin LI, Gjertsen JE, Vinje T, Espehaug B, Fevang JM. Sliding hip screw versus IM nail in reverse oblique trochanteric and subtrochanteric fractures. A study of 2716 patients in the Norwegian hip fracture register. Injury. 2013;44:735-42.

7. Miedel R, Tornkvist H, Ponzer S, Söderqvist A, Tidermark J. Musculoskeletal function and quality of life in elderly patients after a subtrochanteric femoral fracture treated with a cephalomedullary nail. J Ortho Trauma. 2011;25:208-13.

8. Olseen P, Jonsson B, Ceder L, Besjakov J, Olsson O, Sernbo I, et al. The Hansson twin hook is adequate for fixation of trochanteric fractures: 2 fixation failures in a series of 157 prospectively followed patients. Acta Orthop. 2008;79:602-8.

9. Jensen JS, Michaelsen M. Trochanteric femoral fractures treated with McLaughlin osteosynthesis. Acta Orthop Scand. 1975;46:795-803.

10. Seinsheimer F. Subtrochanteric fractures of the femur. J Bone Joint Surg Am. 1978;60:300-6.

11. Baumgaertner MR, Curtin SL, Lindskog DM, Keggi JM. The value of the tipapex distance in predicting failure of fixation of peritrochanteric fractures of the hip. J Bone Joint Surg Am. 1995;77:1058-64.

12. Olsson O, Ceder L, Lunsjo K, Hauggaard A. Biaxial dynamization in unstable intertrochanteric fractures. Good experience with a simplified Medoff sliding plate in 94 patients. Acta Orthop Scand. 1997;68:327-31.

13. Bojan AJ, Beimel C, Taglang G, Collin D, Ekholm C, Jonsson A. Critical factors in cut-out complication after gamma nail treatment of proximal femoral fractures. BMC Musculoskelet Disord. 2013;14:1.

14. Watson JT, Moed BR, Cramer KE, Karges DE. Comparison of the compression hip screw with the Medoff sliding plate for intertrochanteric fractures. Clin Orthop Relat Res. 1998:79-86.

15. Wachtl SW, Gautier E, Jakob RP. Low reoperation rate with the Medoff sliding plate: 1 technical failure in 63 trochanteric hip fractures. Acta Orthop Scand. 2001;72:141-5.

16. McCormack R, Panagiotopolous K, Buckley R, Penner M, Perey B, Pate G, et al. A multicentre, prospective, randomised comparison of the sliding hip screw with the Medoff sliding screw and side plate for unstable intertrochanteric hip fractures. Injury. 2013;44:1904-9.

17. Parker MJ. Trochanteric hip fractures. Fixation failure commoner with femoral medialization, a comparison of 101 cases. Acta Orthop Scand. 1996;67:329-32.

18. Miedel $\mathrm{R}$, Tornkvist $\mathrm{H}$, Ponzer $\mathrm{S}$, Tidermark J. Musculoskeletal function and quality of life after an unstable trochanteric fracture treated with the trochanteric gamma nail. Arch Orthop Trauma Surg. 2012;132:1495-503.

19. Kummer FJ, Olsson O, Pearlman CA, Ceder L, Larsson S, Koval KJ. Intramedullary versus extramedullary fixation of subtrochanteric fractures. A biomechanical study. Acta Orthop Scand. 1998;69:580-4.
20. Mahomed N, Harrington I, Kellam J, Maistrelli G, Hearn T, Vroemen J. Biomechanical analysis of the gamma nail and sliding hip screw. Clin Orthop Relat Res. 1994:280-8.

21. Mahomed MN, Harrington IJ, Hearn TC. Biomechanical analysis of the Medoff sliding plate. J Trauma. 2000;48:93-100.

22. Kouvidis G, Sakellariou VI, Mavrogenis AF, Stavrakakis J, Kampas D, Galanakis J, et al. Dual lag screw cephalomedullary nail versus the classic sliding hip screw for the stabilization of intertrochanteric fractures. A prospective randomized study. Strateg Trauma Limb Reconstr. 2012;7:155-62.

23. Miedel R, Ponzer S, Tornkvist H, Söderqvist A, Tidermark J. The standard gamma nail or the Medoff sliding plate for unstable trochanteric and subtrochanteric fractures. A randomised, controlled trial. J Bone Joint Surg Br. 2005:87:68-75.

24. Ceder L, Lunsjo K, Olsson O, Stigsson L, Hauggaard A. Different ways to treat subtrochanteric fractures with the Medoff sliding plate. Clin Orthop Relat Res. 1998:101-6.

25. Olsson O. Alternative techniques in trochanteric hip fracture surgery. Clinical and biomechanical studies on the Medoff sliding plate and the twin hook. Acta Orthop Scand Suppl. 2000;295:1-31.

26. Lunsjo K, Ceder L, Thorngren KG, Skytting B, Tidermark J, Berntsson PO, et al. Extramedullary fixation of 569 unstable intertrochanteric fractures: a randomized multicenter trial of the Medoff sliding plate versus three other screw-plate systems. Acta Orthop Scand. 2001;72:133-40.

27. Ekstrom W, Karlsson-Thur C, Larsson S, Ragnarsson B, Alberts KA. Functional outcome in treatment of unstable trochanteric and subtrochanteric fractures with the proximal femoral nail and the Medoff sliding plate. J Ortho Trauma. 2007;21:18-25.

28. Swedish Hip Fracture Registry Annual Report 2011. Rikshöft Web Site Available at: http://rikshoft.se/wp-content/uploads/2013/07/arsrapport2011. pdf. Accessed June 2, 2016

29. Palm H, Jacobsen S, Sonne-Holm S, Gebuhr P. Integrity of the lateral femoral wall in intertrochanteric hip fractures: an important predictor of a reoperation. J Bone Joint Surg Am. 2007;89:470-5.

\section{Submit your next manuscript to BioMed Central and we will help you at every step:}

- We accept pre-submission inquiries

- Our selector tool helps you to find the most relevant journal

- We provide round the clock customer support

- Convenient online submission

- Thorough peer review

- Inclusion in PubMed and all major indexing services

- Maximum visibility for your research

Submit your manuscript at www.biomedcentral.com/submit
Biomed Central 